Bull. Korean Math. Soc. 50 (2013), No. 2, pp. 697-704

http://dx.doi.org/10.4134/BKMS.2013.50.2.697

\title{
ON THE SOLUTIONS OF $x^{k}=g$ IN A FINITE GROUP
}

\author{
Sunil Kumar Prajapati and Ritumoni Sarma
}

\begin{abstract}
The function $g \mapsto \zeta_{G}^{k}(g)$ which counts the number of solutions of $x^{k}=g$ in a finite group $G$, is not necessarily a character of $G$. We study this function for the case of dihedral groups and generalized quaternion groups.
\end{abstract}

\section{Introduction}

Let $\mathfrak{F}_{n}$ be the free group on $n$ generators $x_{1}, x_{2}, \ldots, x_{n}$. Suppose that $w\left(x_{1}, x_{2}, \ldots, x_{n}\right) \in \mathfrak{F}_{n}$. For a finite group $G$, define $\zeta_{G}^{w}: G \longrightarrow \mathbb{Z}$ by

$$
\zeta_{G}^{w}(g):=\left|\left\{\left(g_{1}, g_{2}, \ldots, g_{n}\right) \in G^{n}: w\left(g_{1}, g_{2}, \ldots, g_{n}\right)=g\right\}\right| .
$$

We prefer to write $\zeta_{G}^{k}$ instead of $\zeta_{G}^{w}$ if $n=1$ and $w(x)=x^{k}$.

In [1], it was proved that $\zeta_{G}^{k}$ is a generalized character (i.e., a $\mathbb{Z}$-linear combination of irreducible characters). It is easy to prove that $\zeta_{G}^{k}$ is actually a character if $G$ is an abelian group. In fact, for an abelian group $G$, the function $\zeta_{G}^{w}$ is always a character for any $w\left(x_{1}, x_{2}, \ldots, x_{n}\right) \in \mathfrak{F}_{n}$. In general, for a non-abelian group, this function need not be a character. For example, if $Q_{8}$ is the quaternion group, then $\zeta_{Q_{8}}^{2}$ is not a character. In this article, we prove that $\zeta_{G}^{k}$ is a character for finite dihedral groups and generalized quaternion groups except when $k \equiv 2(\bmod 4)$ in the case of generalized quaternion groups.

In [4], it is shown that if $w\left(x_{1}, x_{2}, \ldots, x_{n}\right)=x_{1}^{k_{1}} x_{2}^{k_{2}} \cdots x_{n}^{k_{n}}$, the function $\zeta_{G}^{w}$ is a generalized character for any finite group $G$. Here, Theorem 2.7 provides a sufficient condition for that $\zeta_{G}^{w}$ to be a character.

Throughout the article, $G$ denotes a finite group and $\operatorname{Irr}(G)$, the set of its irreducible characters. For any class functions $f$ and $g$, the expression $\langle f, g\rangle$ denotes their standard inner product. We record an elementary trigonometric

\footnotetext{
Received January 26, 2012; Revised March 30, 2012.

2010 Mathematics Subject Classification. 20C15.

Key words and phrases. finite groups, group characters.

The first author was supported by Council of Scientific and Industrial Research (CSIR),
} India.

(C)2013 The Korean Mathematical Society 
identity for future use.

$$
1+\sum_{1 \leq j \leq n} 2 \cos (j x)=\frac{\sin \left(\left(n+\frac{1}{2}\right) x\right)}{\sin \left(\frac{1}{2} x\right)} .
$$

\section{Main result}

Let $\lambda$ be a generalized character of $G$. For $k \in \mathbb{N}$, define functions $\lambda_{(k)}$ and $\lambda^{(k)}$ from $G$ to $\mathbb{C}$ by

$$
\begin{aligned}
\lambda_{(k)}(g) & :=\sum_{z \in G, z^{k}=g} \lambda(z), \\
\lambda^{(k)}(g) & :=\lambda\left(g^{k}\right) .
\end{aligned}
$$

In [1], it is proved that both $\lambda_{(k)}$ and $\lambda^{(k)}$ are generalized characters of G. The following proposition gives a necessary and sufficient condition for $\zeta_{G}^{k}$ to be a character of $G$.

Proposition 2.1. The function $\zeta_{G}^{k}$ is a character if and only if

$$
c_{\chi}^{(k)}:=\frac{1}{|G|} \sum_{g \in G} \chi\left(g^{k}\right)
$$

is a non-negative integer for every $\chi \in \operatorname{Irr}(G)$.

Proof. If 1 denotes the trivial irreducible character of a group $G$, then by definition

$$
1_{(k)}=\zeta_{G}^{k}
$$

On the other hand, for any two generalized characters $\lambda$ and $\chi$ of $G$, we have

$$
\begin{aligned}
\left\langle\lambda_{(k)}, \chi\right\rangle & =\frac{1}{|G|} \sum_{g \in G} \sum_{z \in G, z^{k}=g} \lambda(z) \chi\left(g^{-1}\right) \\
& =\frac{1}{|G|} \sum_{z \in G} \lambda(z) \chi^{(k)}\left(z^{-1}\right) \\
& =\left\langle\lambda, \chi^{(k)}\right\rangle .
\end{aligned}
$$

In particular, if $\lambda=1$ and $\chi \in \operatorname{Irr}(G)$, we get by $(6)$

$$
\left\langle\zeta_{G}^{k}, \chi\right\rangle=\frac{1}{|G|} \sum_{g \in G} \chi\left(g^{k}\right) .
$$

Hence, the proposition follows.

Now we study $\zeta_{G}^{k}$ when $G$ is the dihedral group $D_{2 n}$ of order $2 n$ with $n \geq 3$. We consider the following presentation: $D_{2 n}=\langle a, b| a^{n}=1, b^{2}=1, b a b^{-1}=$ $\left.a^{-1}\right\rangle$. Then its character table is as follows: 
Table(a): when $n=2 m+1$

\begin{tabular}{|c|ccr|}
\hline$g$ & 1 & $a^{r}(1 \leq r \leq m)$ & $b$ \\
$\left|C_{G}(g)\right|$ & $2 n$ & $n$ & 2 \\
\hline$\chi_{1}$ & 1 & 1 & 1 \\
$\chi_{2}$ & 1 & 1 & -1 \\
$\phi_{j}(1 \leq j \leq m)$ & 2 & $2 \cos \left(\frac{2 \pi j r}{n}\right)$ & 0 \\
\hline
\end{tabular}

Table(b): when $n=2 m$

\begin{tabular}{|ccccrr|}
\hline$g$ & 1 & $a^{m}$ & $a^{r}(1 \leq r \leq(m-1))$ & $b$ & $a b$ \\
$\left|C_{G}(g)\right|$ & $2 n$ & $2 n$ & $n$ & 4 & 4 \\
\hline$\chi_{1}$ & 1 & 1 & 1 & 1 & 1 \\
$\chi_{2}$ & 1 & 1 & 1 & -1 & -1 \\
$\chi_{3}$ & 1 & $(-1)^{m}$ & $(-1)^{r}$ & 1 & -1 \\
$\chi_{4}$ & 1 & $(-1)^{m}$ & $(-1)^{r}$ & -1 & 1 \\
$\phi_{j}(1 \leq j \leq(m-1))$ & 2 & $2(-1)^{j}$ & $2 \cos \left(\frac{2 \pi j r}{n}\right)$ & 0 & 0 \\
\hline
\end{tabular}

Lemma 2.2. Let $n \geq 3$. Suppose that $\chi$ is a nonlinear irreducible character of $D_{2 n}$. With the notation in the preceding paragraph, for every $k \in \mathbb{N}$, we have

$$
\sum_{1 \leq r \leq n} \chi\left(a^{r k}\right)=\left\{\begin{array}{lc}
2 n & \text { if } n \mid k, \\
0 & \text { otherwise. }
\end{array}\right.
$$

Proof. If $n \mid k$, then for any nonlinear irreducible character $\chi=\phi_{j}$ (see Table(a) and Table(b)) of $D_{2 n}$, we have

$$
\sum_{1 \leq r \leq n} \phi_{j}\left(a^{r k}\right)=\sum_{1 \leq r \leq n} \phi_{j}(1)=2 n .
$$

Next assume that $n \nmid k$. Let $d:=\operatorname{gcd}(n, k)$ and $\chi=\phi_{j}$. Then

$$
\sum_{1 \leq r \leq n} \phi_{j}\left(a^{r k}\right)=\sum_{1 \leq r \leq \frac{n}{d}} d \cdot \phi_{j}\left(a^{r d}\right) .
$$

If $n=2 m+1$, then (7) becomes

$$
\begin{aligned}
d \cdot \phi_{j}(1)+\sum_{1 \leq r \leq\left(\frac{n}{d}-1\right) / 2} 2 d \cdot \phi_{j}\left(a^{r d}\right) & =2 d+2 d \sum_{1 \leq r \leq\left(\frac{n}{d}-1\right) / 2} 2 \cos \left(r \frac{2 \pi j d}{n}\right) \\
& =2 d+2 d \cdot(-1) \quad(\text { by using }(2)) \\
& =0 .
\end{aligned}
$$

Next, suppose that $n=2 m$. If $n / d$ is odd, then $r d \neq m$ for $1 \leq r \leq\left(\frac{n}{d}-1\right) / 2$. Thus, the computation of (7), is exactly like that of the case $n=2 m+1$. Finally, if $n / d$ is even, (7) reduces to

$$
d \cdot \phi_{j}(1)+d \cdot \phi_{j}\left(a^{m}\right)+\sum_{1 \leq r \leq\left(\frac{n}{d}-2\right) / 2} 2 d \cdot \phi_{j}\left(a^{r d}\right)
$$




$$
\begin{aligned}
& =2 d+2 d \cdot(-1)^{j}+2 d \sum_{1 \leq r \leq\left(\frac{n}{d}-1\right) / 2} 2 \cos \left(r \frac{2 \pi j d}{n}\right) \\
& \left.=2 d+2 d \cdot(-1)^{j}+2 d \cdot\left\{(-1)^{j-1}-1\right\} \text { (by using }(2)\right) \\
& =0 .
\end{aligned}
$$

This completes the proof of the lemma.

Theorem 2.3. For every $k, n \in \mathbb{N}$, the function $\zeta_{D_{2 n}}^{k}$ is a character.

Proof. If $n \leq 2, D_{2 n}$ is an abelian group and hence $\zeta_{D_{2 n}}^{k}$ is a character for any $k \in \mathbb{N}$. Now for $n \geq 3$, by Proposition 2.1 , it is sufficient to show that $c_{\chi}^{(k)} \geq 0$ for each $\chi \in \operatorname{Irr}\left(D_{2 n}\right)$.

First we deal with the nonlinear characters $\phi_{j}$ (see Table(a) and Table(b)). Case $(n=2 m+1)$ : by Lemma 2.2 ,

$$
c_{\phi_{j}}^{(k)}=\frac{1}{\left|D_{2 n}\right|} \sum_{g \in D_{2 n}} \phi_{j}\left(g^{k}\right)= \begin{cases}1+\alpha_{j}(a, k) & \text { if } n \mid k, \\ \alpha_{j}(a, k) & \text { otherwise }\end{cases}
$$

where $\alpha_{j}(a, k)=\frac{1}{2 n}\left\{\left|C l_{D_{2 n}}(b)\right| \cdot \phi_{j}\left(b^{k}\right)\right\}$. If $k$ is even, $b^{k}=1$. Then, we have

$$
\alpha_{j}(a, k)=\frac{1}{2 n}\left\{n \cdot \phi_{j}(1)\right\}=1 .
$$

If $k$ is odd, $b^{k}=b$. Then, $\alpha_{j}(a, k)=0$. Hence in either cases, by $(8), c_{\phi_{j}}^{(k)}$ is a positive integer.

Case $(n=2 m)$ : by Lemma 2.2 ,

$$
c_{\phi_{j}}^{(k)}=\frac{1}{\left|D_{2 n}\right|} \sum_{g \in D_{2 n}} \phi_{j}\left(g^{k}\right)= \begin{cases}1+\beta_{j}(b, k) & \text { if } n \mid k, \\ \beta_{j}(b, k) & \text { otherwise }\end{cases}
$$

where $\beta_{j}(b, k)=\frac{1}{2 n}\left\{\left|C l_{D_{2 n}}(b)\right| \cdot \phi_{j}\left(b^{k}\right)+\left|C l_{D_{2 n}}(a b)\right| \cdot \phi_{j}\left((a b)^{k}\right)\right\}$. If $k$ is even, $b^{k}=(a b)^{k}=1$. Therefore, we have

$$
\beta_{j}(b, k)=\frac{1}{2 n}\left\{m \cdot \phi_{j}(1)+m \cdot \phi_{j}(1)\right\}=1 .
$$

If $k$ is odd, $b^{k}=b$ and $(a b)^{k}=a b$. Then, $\beta_{j}(b, k)=0$. Hence in both cases, by $(9), c_{\phi_{j}}^{(k)}$ is a positive integer.

Finally, for a linear character $\chi$, it is not difficult to show that $c_{\chi}^{(k)}$ is either zero or one. Indeed, if $n=2 m+1, c_{\chi_{1}}^{(k)}=1$ and $c_{\chi_{2}}^{(k)}=1$ or 0 depending upon whether $k$ is even or odd. Similarly, if $n=2 m$, then $c_{\chi_{1}}^{(k)}=1, c_{\chi_{i}}^{(k)}=1$ or 0 according as $k$ is even or odd for each $i=2,3,4$. This completes the proof.

Next we study $\zeta_{G}^{k}$ when $G$ is the generalized quaternion group $Q_{2 n}$ of order $4 n$ with $n \geq 2$. We consider the following presentation: $Q_{2 n}=\left\langle a, b: a^{2 n}=\right.$ $\left.1, a^{n}=b^{2}, b a b^{-1}=a^{-1}\right\rangle$. When $n$ is odd, the character table of $Q_{2 n}$ is given by Table(c). When $n$ is even, the character table of $Q_{2 n}$ is obtained from Table(c) by replacing $i$ by 1 . 
Table(c)

\begin{tabular}{|c|cccrr|}
\hline$g$ & 1 & $a^{n}$ & $a^{r}(1 \leq r \leq(n-1))$ & $b$ & $a b$ \\
$\left|C_{G}(g)\right|$ & $4 n$ & $4 n$ & $2 n$ & 4 & 4 \\
\hline$\chi_{1}$ & 1 & 1 & 1 & 1 & 1 \\
$\chi_{2}$ & 1 & 1 & 1 & -1 & -1 \\
$\chi_{3}$ & 1 & $(-1)^{n}$ & $(-1)^{r}$ & $i$ & $-i$ \\
$\chi_{4}$ & 1 & $(-1)^{n}$ & $(-1)^{r}$ & $-i$ & $i$ \\
$\phi_{j}(1 \leq j \leq(n-1))$ & 2 & $2(-1)^{j}$ & $2 \cos \left(\frac{\pi j r}{n}\right)$ & 0 & 0 \\
\hline
\end{tabular}

Lemma 2.4. Let $n \geq 3$. Suppose that $\chi$ is a nonlinear irreducible character of $Q_{2 n}$. With the notation in the preceding paragraph, for every $k \in \mathbb{N}$ we have

$$
\sum_{1 \leq r \leq 2 n} \chi\left(a^{r k}\right)= \begin{cases}4 n & \text { if } 2 n \mid k, \\ 0 & \text { otherwise. }\end{cases}
$$

Proof. If $2 n \mid k$, then for any nonlinear irreducible character $\chi=\phi_{j}$ (see Table(c)) of $Q_{2 n}$, we have

$$
\sum_{1 \leq r \leq 2 n} \phi_{j}\left(a^{r k}\right)=\sum_{1 \leq r \leq 2 n} \phi_{j}(1)=4 n .
$$

Next assume that $2 n \nmid k$. Let $d:=\operatorname{gcd}(2 n, k)$ and $\chi=\phi_{j}$. If $2 n / d$ is odd, then

$$
\begin{aligned}
\sum_{1 \leq r \leq 2 n} \phi_{j}\left(a^{r k}\right) & =d \cdot \phi_{j}(1)+\sum_{1 \leq r \leq\left(\frac{2 n}{d}-1\right) / 2} 2 d \cdot \phi_{j}\left(a^{r d}\right) \\
& =2 d+2 d \sum_{1 \leq r \leq\left(\frac{2 n}{d}-1\right) / 2} 2 \cos \left(r \frac{d j \pi}{n}\right) \\
& =2 d+2 d(-1)(\text { by using }(2)) \\
& =0 .
\end{aligned}
$$

If $2 n / d$ is even, then

$$
\begin{aligned}
\sum_{1 \leq r \leq 2 n} \phi_{j}\left(a^{r k}\right) & =d \cdot \phi_{j}(1)+\sum_{1 \leq r \leq\left(\frac{2 n}{d}-2\right) / 2} 2 d \cdot \phi_{j}\left(a^{r d}\right)+d \phi_{j}\left(a^{n}\right) \\
& =2 d+2 d \sum_{1 \leq r \leq\left(\frac{2 n}{d}-2\right) / 2} 2 \cos \left(r \frac{d j \pi}{n}\right)+2 d(-1)^{j} \\
& =2 d+2 d\left\{(-1)^{j-1}-1\right\}+2 d(-1)^{j} \text { (by using (2)) } \\
& =0 .
\end{aligned}
$$

This completes the proof of the lemma.

Theorem 2.5. For every $n \in \mathbb{N}$, the function $\zeta_{Q_{2 n}}^{k}$ is a character for $k \equiv 0,1$ or $3(\bmod 4)$. When $k \equiv 2(\bmod 4), \zeta_{Q_{2 n}}^{k}$ is a character if and only if $2 n \mid k$. 
Proof. We compute $c_{\chi}^{(k)}$ for each $\chi \in \operatorname{Irr}\left(Q_{2 n}\right)$. First consider the nonlinear irreducible characters $\phi_{j}(1 \leq j<n)$ (see Table(c)). By Lemma 2.4, we have

$$
c_{\phi_{j}}^{(k)}= \begin{cases}1+\alpha_{j}(b, k) & \text { if } 2 n \mid k, \\ \alpha_{j}(b, k) & \text { otherwise }\end{cases}
$$

where $\alpha_{j}(b, k)=\frac{1}{4 n}\left\{\left|C l_{Q_{2 n}}(b)\right| \cdot \phi_{j}\left(b^{k}\right)+\left|C l_{Q_{2 n}}(a b)\right| \cdot \phi_{j}\left((a b)^{k}\right)\right\}$. We perform the computation in four exhaustive cases.

Case $k \equiv 2(\bmod 4)$ : Then $b^{k}=(a b)^{k}=a^{n}$. Therefore,

$$
\begin{aligned}
\alpha_{j}(b, k) & =\frac{1}{4 n}\left\{n \cdot \phi_{j}\left(a^{n}\right)+n \cdot \phi_{j}\left(a^{n}\right)\right\} \\
& =(-1)^{j} .
\end{aligned}
$$

Hence $c_{\phi_{1}}^{(k)}=-1$, under the condition $k \equiv 2(\bmod 4)$ and $2 n \nmid k$.

Case $k \equiv 0(\bmod 4)$ : Then $b^{k}=(a b)^{k}=1$. Therefore,

$$
\begin{aligned}
\alpha_{j}(b, k) & \left.=\frac{1}{4 n}\left\{n \cdot \phi_{j}(1)+n \cdot \phi_{j}(1)\right)\right\} \\
& =1 .
\end{aligned}
$$

Case $k \equiv 1(\bmod 4):$ Then $b^{k}=b,(a b)^{k}=a b$. Therefore, we have

$$
\begin{aligned}
\alpha_{j}(b, k) & =\frac{1}{4 n}\left\{n \cdot \phi_{j}(b)+n \cdot \phi_{j}(a b)\right\} \\
& =0 .
\end{aligned}
$$

Case $k \equiv 3(\bmod 4)$ : Then $b^{k}=a^{n} b$ and $(a b)^{k}=a^{n+1} b$. Therefore they are conjugate to either $b$ or $a b$. Hence $\alpha_{j}(b, k)=0$.

Hence by $(10), c_{\phi_{j}}^{(k)} \geq 0$ for all nonlinear irreducible character of $Q_{2 n}$ except the case when $k \equiv 2(\bmod 4)$ and $2 n \nmid k$.

Finally, for the linear characters of $Q_{2 n}$ we have $c_{\chi_{1}}^{(k)}=1 ; c_{\chi_{2}}^{(k)}=1$ or 0 according as $k$ is even or odd and $c_{\chi_{3}}^{(k)}=c_{\chi_{4}}^{(k)}=0$ or 1 according as $(k-1)(n-1)$ is even or odd.

Thus, when $k \not \equiv 2(\bmod 4)$ or $2 n \mid k, c_{\chi}^{(k)} \geq 0$ for each $\chi \in \operatorname{Irr}\left(Q_{2 n}\right)$. Hence, the theorem follows from Proposition 2.1.

We have the following theorem for the symmetric group $S_{n}$ of degree $n$.

Theorem 2.6. For every $n \geq 1$, the function $\zeta_{S_{n}}^{2}$ is a character.

Proof. By Proposition 2.1, it is sufficient to show that $c_{\chi}^{(2)}$ is a non-negative integer. Since every irreducible character of $S_{n}$ is defined over the real field [2, theorem 75.19], the theorem follows from [3, Corollary 4.15 ].

Theorem 2.7. Let $S$ be a nonempty subset of $\mathbb{Z}$. Suppose that $\zeta_{G}^{k}$ is a character of $G$ for every $k \in S$. If $k_{1}, k_{2}, \ldots, k_{n} \in S$, then $\zeta_{G}^{w}$ is a character of $G$ for $w\left(x_{1}, x_{2}, \ldots, x_{n}\right)=x_{1}^{k_{1}} x_{2}^{k_{2}} \cdots x_{n}^{k_{n}}$. 
Table(d)

\begin{tabular}{|c|rrrrr|}
\hline$g$ & 1 & $(12)$ & $(12)(34)$ & $(123)$ & $(1234)$ \\
\hline$\chi_{1}$ & 1 & 1 & 1 & 1 & 1 \\
$\chi_{2}$ & 1 & -1 & 1 & -1 & 1 \\
$\chi_{3}$ & 3 & 1 & -1 & 0 & -1 \\
$\chi_{4}$ & 3 & -1 & -1 & 0 & 1 \\
$\chi_{5}$ & 2 & 0 & 2 & -1 & 0 \\
\hline
\end{tabular}

Proof. The proof is by induction on $n$. By assumption, the statement holds for $n=1$. Suppose that $n \geq 2$. Let $u\left(x_{1}, x_{2}, \ldots, x_{n-1}\right)=x_{1}^{k_{1}} x_{2}^{k_{2}} \cdots x_{n-1}^{k_{n-1}}$. Then $w=u \cdot x_{n}^{k_{n}}$. By assumption, $\zeta_{G}^{k_{n}}$ is a character and by the induction hypothesis, $\zeta_{G}^{u}$ is a character. Therefore, we may assume that for some $\alpha_{\chi}, \beta_{\chi} \in \mathbb{N} \cup\{0\}$,

$$
\zeta_{G}^{k_{n}}=\sum_{\chi \in \operatorname{Irr}(G)} \alpha_{\chi} \chi, \zeta_{G}^{u}=\sum_{\chi \in \operatorname{Irr}(G)} \beta_{\chi} \chi
$$

Then we have,

$$
\begin{aligned}
\zeta_{G}^{w}(g) & =\sum_{x_{1}, x_{2}, \ldots, x_{n-1} \in G} \zeta_{G}^{k_{n}}\left(\left(x_{1}^{k_{1}} \cdots x_{n-2}^{k_{n-2}} x_{n-1}^{k_{n-1}}\right)^{-1} g\right) \\
& =\sum_{t \in G} \zeta_{G}^{k_{n}}\left(t^{-1} g\right) \zeta_{G}^{u}(t) \\
& =\sum_{\chi, \psi \in \operatorname{Irr}(G)} \alpha_{\chi} \beta_{\psi} \sum_{t \in G} \chi\left(t^{-1} g\right) \psi(t)(\text { using }(11)) \\
& =\sum_{\chi \in \operatorname{Irr}(G)} \alpha_{\chi} \beta_{\chi} \frac{|G|}{\chi(1)} \chi(g) \text { (by orthogonality relation [3, Theorem 2.13 ]). }
\end{aligned}
$$

Since the coefficient of $\chi$ in $\zeta_{G}^{w}$ is non-negative for each $\chi \in \operatorname{Irr}(G)$, the function $\zeta_{G}^{w}$ is a character.

Corollary 2.8. Suppose that $w\left(x_{1}, x_{2}, \ldots, x_{n}\right)=x_{1}^{k_{1}} x_{2}^{k_{2}} \cdots x_{n}^{k_{n}}$. Then

(1) The function $\zeta_{D_{2 n}}^{w}$ is a character.

(2) The function $\zeta_{Q_{2 n}}^{w}$ is a character if $k_{i} \equiv 0,1$ or $3(\bmod 4)$ for each $i$.

Remark 2.9. If the hypothesis of Theorem 2.7 is weakened by allowing repetition of letters in $w$, then the conclusion is no more valid. For example, if $S=\{ \pm 1, \pm 2\}$ and $w(x, y, z)=x^{2} y z x^{-1} z^{-1} y^{-1}$, the function $\zeta_{S_{4}}^{w}$ is not a character (although $\zeta_{S_{4}}^{ \pm 1}, \zeta_{S_{4}}^{ \pm 2}$ are characters). In fact,

$$
\zeta_{S_{4}}^{w}=1152 \chi_{1}+0 \chi_{2}+192 \chi_{3}-192 \chi_{4}+288 \chi_{5},
$$

where $\chi_{1}, \chi_{2}, \chi_{3}, \chi_{4}$ and $\chi_{5}$ are the irreducible characters of $S_{4}$ defined in Table(d). 


\section{References}

[1] R. M. Bryant and L. G. Kovács, A note on generalized characters, Bull. Aust. Math. Soc. 5 (1971), no. 2, 265-269.

[2] C. W. Charles and I. Reiner, Methods of Representation Theory. Vol. II, With applications to finite groups and orders, John Wiley \& Sons Inc., New York, 1987.

[3] I. M. Isaacs, Character Theory of Finite Groups, AMS Chelsea Publishing, Academic Press, New York, 2000.

[4] A. Kerber and B. Wagner, Gleichungen in endlichen Gruppen, Arch. Math. (Basel) 35 (1980), no. 3, 252-262.

Sunil Kumar Prajapati

Department of Mathematics

Indian Institute of Technology Delhi

Hauz Khas, New Delhi-110016, India

E-mail address: skprajapati.iitd@gmail.com

Ritumoni SARMA

Department of Mathematics

Indian Institute of Technology Delhi

Hauz Khas, New Delhi-110016, India

E-mail address: ritumoni@maths.iitd.ac.in 